\title{
Effect of a Dissipative Medium of Finite Size on Antenna Measurement
}

\author{
K. Iizuka and R. W. P. King \\ Contribution from Gordon McKay Laboratory, Harvard University, Cambridge 38, Mass.
}

(Received December 7, 1962; revised January 11, 1963)

\begin{abstract}
An experimental investigation was made of the driving-point admittance of a dipole antenna immersed in a finite dissipative medium in order to obtain fundamental information about the dependence of the admittance upon the size of the medium. This information is intended to assist in determining the size of a tank filled with a dissipative medium for use in studying the properties of antennas in such a finite medium.

The effect of reflections from the side and back walls was investigated quantitatively by means of sixteen tanks of different sizes. It was shown that a shift in the position of an antenna from the center of the tank is equivalent to enlarging the dimensions of the tank. A resonance phenomenon was observed and studied in terms of (a) the distortion of the admittance curve as a function of the size of the tank, (b) the distribution of the current on the antenna, (c) the frequency characteristic of the field intensity inside the tank, and (d) the sensitivity of the system to perturbation. Certain precautions to be taken in the design of a dissipative-solution-filled tank are discussed briefly.
\end{abstract}

\section{Introduction}

Interest in the characteristics of antennas immersed in various conducting media has increased in recent years because of possible application to antennas under a landing strip for aircraft, probe antennas for geophysical exploration, submarine antennas, missile antennas in plasmas, and of quite recent concern, buried antennas for survival telecommunications. Since in most cases theoretical analyses are complicated and numerical calculations are invariably difficult even in the simplest cases, experimental investigations have a special significance. It is a general practice to construct a tank of finite size as an approximation of a medium that is infinite in extent. The tank is filled with a substance whose conductivity can be changed by simply adding measured quantities of suitable materials. In most cases water solutions of sodium chloride are conveniently used as a conducting dielectric, $\epsilon=78 \sim 70$ at $114 \mathrm{Mc} / \mathrm{s}$ [Tizuka, 1963a, 1963b; Iizuka and King, 1961a, 1961b, 1962a, 1962b, and 1962c; King and Iizuka, 1963]. The conductivity is easily controlled by the percentage of sodium chloride. For example, the dimensionless ratio $\sigma / \omega \epsilon_{r} \epsilon_{0}$ may be allowed to range from 0.036 to 8.8 at $114 \mathrm{Mc} / \mathrm{s}$. Practically speaking, it is preferable to choose a \footnotetext{
1 The research reported in this paper was made possible through support ex-
tended Cruft Laboratory, Harvard University, by the Geophysics Research tended Cruft Laboratory, Harvard University, by the Geophysics Research
Directorate, U.S. Air Force, under Contract A F19(604)-7262 and by the Nationa! Science Foundation under Grant 20225.
}

liquid with a moderately low dielectric constant that has the property of dissolving suitable compounds in order to change the conductivity of the solution. When the dielectric constant is low, the wavelength is not unreasonably short, so that the accurate measurement of the current amplitude and phase distributions along a submerged antenna are not difficult. Moreover the wavelength is not inconveniently long in the air- or plastic-filled coaxial line which is used to measure the driving-point admittance. One of the better candidates is dioxane $\mathrm{C}_{4} \mathrm{H}_{8} \mathrm{O}_{2} \quad\left(\epsilon_{r} \sim 2.21, \quad \sigma=5 \times 10^{-15} \mathrm{mho}^{-\mathrm{cm}^{-1}}\right.$ at low frequencies and the value of $\epsilon_{r}$ becomes slightly smaller at higher frequencies) which has the ability to dissolve the compound tetramethyl-ammonium $\left(\mathrm{CH}_{3}\right)_{4}$ $\mathrm{NOH}$ with which the conductivity of the solution may be varied. However, dioxane would require special precautions in designing components, because it readily dissolves most plastic materials and is toxic as well as flammable.

How close are the results measured in a tank of finite size to those measured in a medium that is infinite in extent? To the best of the author's knowledge, no quantitative answer to this question has been given, and it is to provide such an answer that measurements have been made of the drivingpoint admittance of an antenna immersed in a series of fluid-filled tanks of different sizes.

In the representation of theoretical and experimental results, the skin depth is often used as the 
dependent variable in graphical representations of the properties of antennas immersed in dissipative media. Actually, this is a convenient variable primarily when highly conducting solutions and plane waves are involved. Since the finite size of a tank is a greater limitation when the solution is not highly conducting, the wavelength is here preferred as the variable.

The electromagnetic field of a thin half-wave dipole in an infinite dissipative medium consists essentially of expanding spheroidal wave fronts similar to those in air, but with an added exponential attenuation. Complete formulas and discussions are in the literature [King, 1961]. In a medium of finite size, similarly attenuated reflections from the walls combine with this direct field to generate a highly complicated total field. This reflected field may produce significant differences in the distribution of the current and the impedance of the antenna.

\section{Experimental Ârrangement}

For the purpose of the present investigation, the previously described apparatus designed for the study of antennas in a homogeneous dissipative medium [Iizuka and King, 1961a, 1962a] was used with certain modifications. The original tank had the dimensions $4 \frac{1}{2} \lambda_{w}, 4 \frac{1}{2} \lambda_{w}$, and $2 \frac{5}{8} \lambda_{w}$, where $\lambda_{w}=$ $29.77 \mathrm{~cm}$ is the wavelength in the water solution. The corresponding frequency is $114 \mathrm{Mc} / \mathrm{s}$. Five of the six walls are wooden, the sixth, arranged in a vertical position, is an aluminum ground plane with dimensions $4 \frac{1}{2} \lambda_{w}$ and $4 \frac{1}{2} \lambda_{w}$. This latter is pierced by the dielectric and the inner conductor of a horizontal coaxial line which extends into the solution. The largest tank is conveniently called the main tank to distinguish it from the sixteen smaller tanks which were sucessively installed in it.

The driving-point admittance of the monopole was obtained by measuring the standing-wave pattern on the coaxial line with a movable current probe. A cutout view of the experimental setup is shown in figure 1.

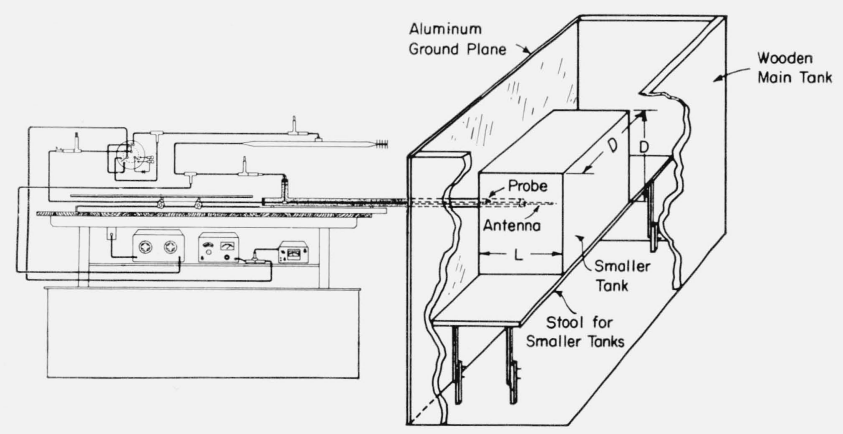

Figure 1. Cut-away view of the experimental setup for measuring the driving-point admittance with various sizes of tanks.

\section{Reflections From the Tank Walls}

First, consider the simple case of an incident plane wave impinging from a water-filled half-space perpendicularly on an infinite wooden wall in the $x y$ plane. Behind the wall is air. The problem is one of reflection from the boundaries of the three parallel dielectrics with two discontinuities of the medium. The geometry is shown in figure 2a. The impedance $Z_{i 2}$ at the boundary between the solution and the wooden wall looking to the right in figure $2 \mathrm{a}$ may be expressed in terms of the propagation constant, $k_{2}=\alpha+j \beta_{2}$ of the wood, the wave impedance $\eta_{2}$ of the solution, the wave impedance $\eta_{3}$ of the air and the thickness $l$ of the wooden wall, when the solution and the wooden walls are considered to be perfect dielectrics as follows [Ramo and Whinnery, 1953]:

$$
Z_{i 2}=\eta_{2}\left[\frac{\eta_{3} \cos \beta_{2} l+j \eta_{2} \sin \beta_{2} l}{\eta_{2} \cos \beta_{2} l+j \eta_{3} \sin \beta_{2} l}\right]
$$

When $\beta_{2} l$ is much smaller than one, $Z_{i 2}$ can be approximated by

$$
Z_{i 2} \simeq \eta_{3}\left[1+j \beta_{2} l\left(\frac{\eta_{2}}{\eta_{3}}-\frac{\eta_{3}}{\eta_{2}}\right)\right]
$$

The reflection coefficient $\rho$ at the solution-wood surface is

$$
\rho=\frac{Z_{i 2}-\eta_{1}}{Z_{i 2}+\eta_{1}}
$$

If (2) is inserted into (3), the result is

$$
\rho=\frac{\left[1+j \beta_{2} l\left(\frac{\eta_{2}}{\eta_{3}}-\frac{\eta_{3}}{\eta_{2}}\right)\right]-\frac{\eta_{1}}{\eta_{3}}}{\left[1+j \beta_{2} l\left(\frac{\eta_{2}}{\eta_{3}}-\frac{\eta_{3}}{\eta_{2}}\right)\right]+\frac{\eta_{1}}{\eta_{3}}} .
$$

In the particular case when the solution and the wooden walls are approximated by perfect dielectrics, the effects of the reflections from the walls are most serious. In this case the value of $|\rho|$ in (4) is calculated to be 0.78 when the thickness of the wooden walls of the actual tank is inserted in the equation. Thus, more than half of the incident power is reflected back toward the source $\left(|\rho|^{2}=0.62\right)$, and the standing-wave ratio for plane waves as calculated with this value of $\rho$ is 8.1. Actually, the incident wave is not plane, but roughly spheroidal, so that the reflected wave diverges from the plane of discontinuity. Also, neither medium is a perfect dielectric. Hence, the actual values of $|\rho|$ and of the standing-wave ratio should be smaller than those obtained above and the results calculated from (4) give an idea of the worst case to be expected.

Measurements of the standing-wave pattern in the finite tank were made to determine the actual situation. A solution with $\frac{\sigma}{\omega \epsilon_{r} \epsilon_{0}}=0.0354$ where $\sigma$ is the conductivity of the solution was used. This is the smallest value of $\frac{\sigma}{\omega \epsilon_{r} \epsilon_{0}}$ obtainable with tap water at $114 \mathrm{Mc} / \mathrm{s}$ and it is the smallest value used 


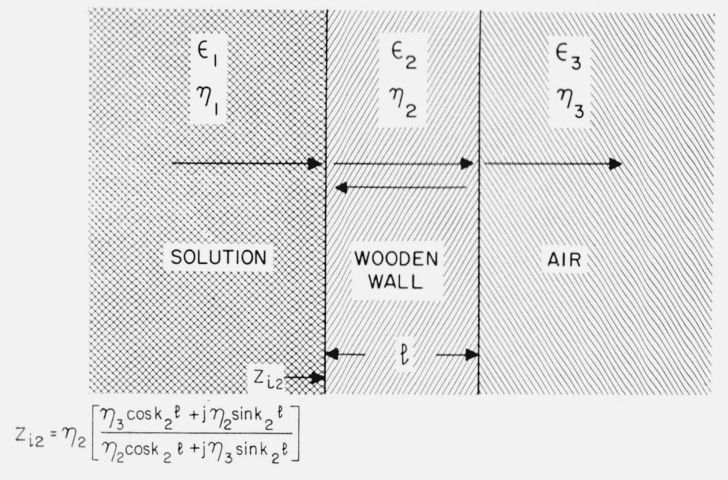

Figure 2a. Reflection from the tank wall.

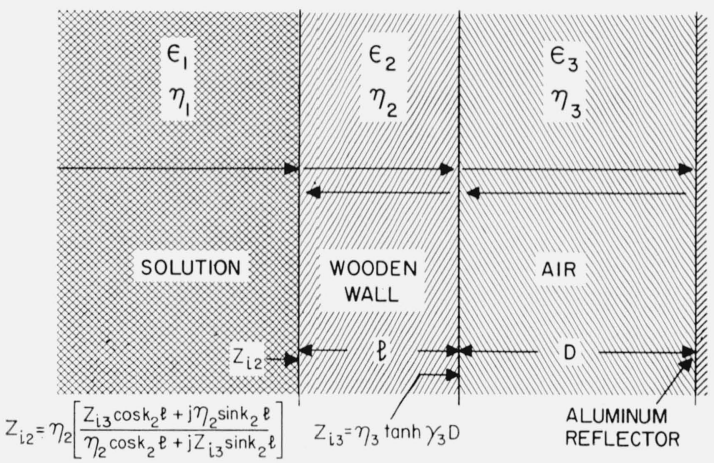

Figure 2b. Reflection from the tank wall with reflector.

in most of the measurements of the driving-point admittance of antennas [Iizuka, 1963a; Iizuka and King, 1961a]. Hence, the effect of wall reflections on the driving-point admittance is greatest for this value of $\frac{\sigma}{\omega \epsilon_{r} \epsilon_{0}}$. A shielded-loop probe was moved along the ground plane horizontally from the position of the antenna to the edge of the tank wall. The output from the probe is shown in figure 3 together with the corresponding magnetic field distribution $H_{\phi}$ in a dissipative medium of infinite size as obtained by King [1961]. It is seen from the figure that the distribution of the field intensity inside the tank more or less follows the theoretical curve of the spheroidal wave except for periodic fluctuations due to the reflected wave from the wall. A rough measure of the relative amplitude of the standing wave may be obtained by averaging the ratio of maximum to next-adjacent minimum over the part of the pattern for which the ideal theoretical curve is reasonably flat. The value so obtained is 1.33 . This is much smaller than the SWR obtained with the assumption that the incident wave is a plane wave and the media are perfect dielectrics infinite in the $x$ and $y$ directions. Nevertheless, the question arises what effect a standing wave of this amplitude has on the measured values of the driving-point admittance. This problem is studied further in the following sections.

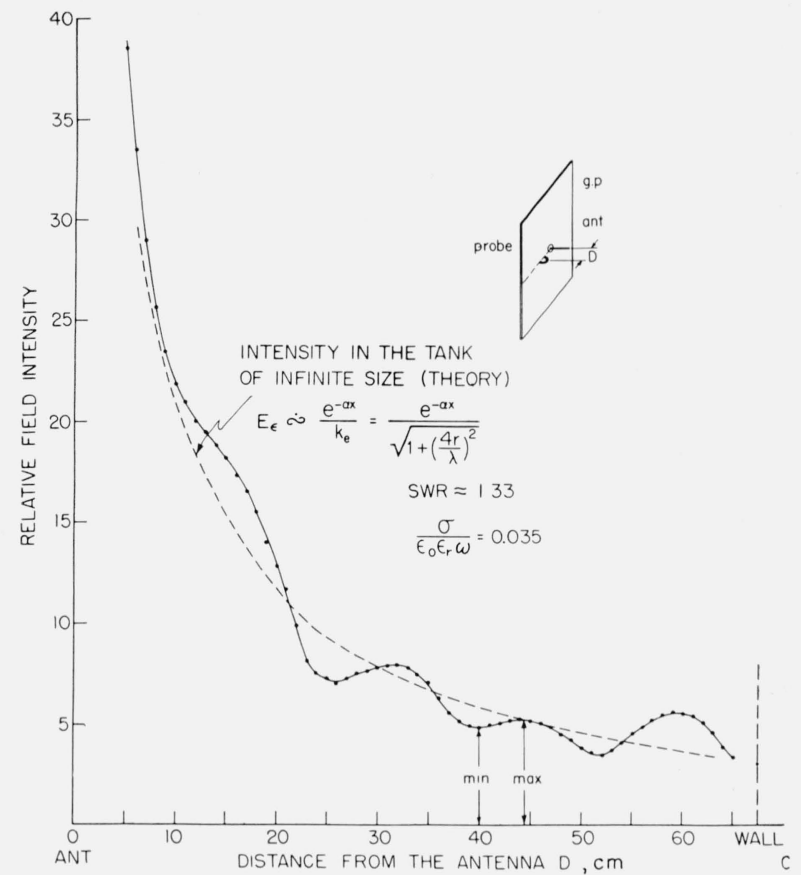

Figure 3. Field distribution in the tank when $S W R=1.33$

\section{Driving-Point Admittance as a Function of Different Reflections From the Walls}

The effect of different reflections from the walls of the main tank on the driving-point admittance of an antenna was determined when the five wooden sides of the tank were backed by aluminum screens as shown in figure $2 \mathrm{~b}$ and figure 4 . The spacing $D$, between the aluminum screen and the tank walls was varied, and with it the impedance $Z_{i 3}$ looking from the outer surface of the tank wall towards the aluminum screen. For infinite plane surfaces and plane waves this latter is given by

$$
Z_{i 3}=j \eta_{3} \tan \beta_{3} D
$$

where $\beta_{3}$ is the propagation constant of the air. Evidently $Z_{i 3}$ can be changed practically from zero to infinity by varying $D$. A change in $Z_{i 3}$ affects $Z_{i 2}$ according to the relation

$$
Z_{i 2}=\eta_{2}\left[\frac{Z_{i 3} \cos \beta_{2} l+j \eta_{2} \sin \beta_{2} l}{\eta_{2} \cos \beta_{2} l+j Z_{i 3} \sin \beta_{2} l}\right] .
$$

The value of $Z_{i 2}$ is related to the plane-wave reflection coefficient $\rho$ by (3). Although these plane-wave expressions are not applicable to the finite tank, they indicate that a change in $D$ should cause a significant change in the reflection from the walls of the tank.

The measured driving-point admittance of an antenna with $\beta h=\frac{2 \pi}{\lambda_{w}} h=1.78$ immersed in a solution with $\epsilon_{r}=78$ and $\frac{\sigma}{\omega \epsilon_{r} \epsilon_{0}}=0.0354$ is tabulated in table 1 as a function of $D$. It is seen that the driving- 


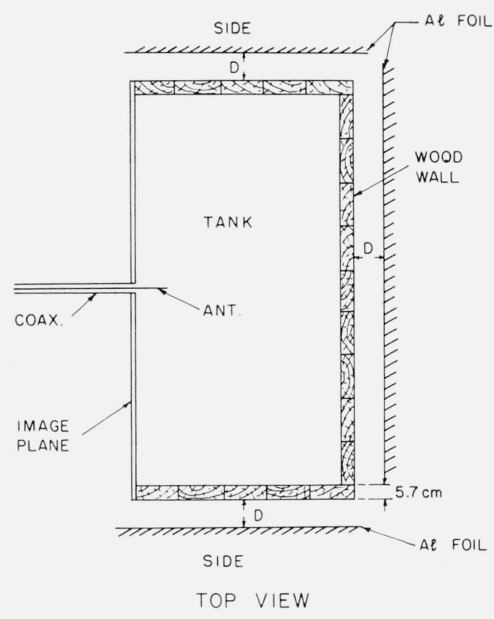

Figure 4. Geometry of aluminum reflector.

point admittance varies only slightly with changes in $D$. Hence, it may be concluded that an error in the measurement of the driving-point admittance due to reflections from the walls of the main tank is very small, and the size of the main tank seems to be adequate. However, the question naturally arises: Could a tank of smaller size which would be more economical to construct as well as easier to handle, be used? If so, what factors determine the minimum size of a tank for a given error in the drivingpoint admittance of an antenna?

TABLE 1. Driving-point admittance of a dipole antenna with $\beta \mathrm{h}=1.78$ in the solution with $\epsilon_{r}=78, \frac{\sigma}{\omega \epsilon_{r} \epsilon_{o}}=0.0354$ with aluminum reflectors placed outside the tank on five sides at distance D

\begin{tabular}{|c|c|c|}
\hline$D$ & $G$ & $B$ \\
\hline $\begin{array}{l}\text { Without screen } \\
0.022 \lambda_{\text {air }} \\
.092 \lambda_{\text {air }} \\
.117 \lambda_{\text {air }} \\
.172 \lambda_{\text {air }} \\
.235 \lambda_{\text {air }} \\
\text { Back wall only a }\end{array}$ & $\begin{array}{r}\text { Millimhos } \\
32.4 \\
31.3 \\
32.9 \\
34.4 \\
35.0 \\
33.9 \\
32.9\end{array}$ & $\begin{array}{r}\text { Millimhos } \\
23.0 \\
21.9 \\
21.7 \\
23.0 \\
24.0 \\
25.1 \\
24.5\end{array}$ \\
\hline
\end{tabular}

a All the side reflectors were removed and only the reflector at the back wall retained.

\section{Significance of the Dimension Between the Side Walls}

Sixteen smaller wooden tanks with progressively increasing widths as shown in figure 5 were arranged for successive installation inside the main tank. They were numbered from 1 for the smallest, to 16 for the largest. Each small tank rested on a tripod of adjustable height as shown in figure 1, and its open face was sealed to the ground plane by means of suitably arranged screws and gaskets.

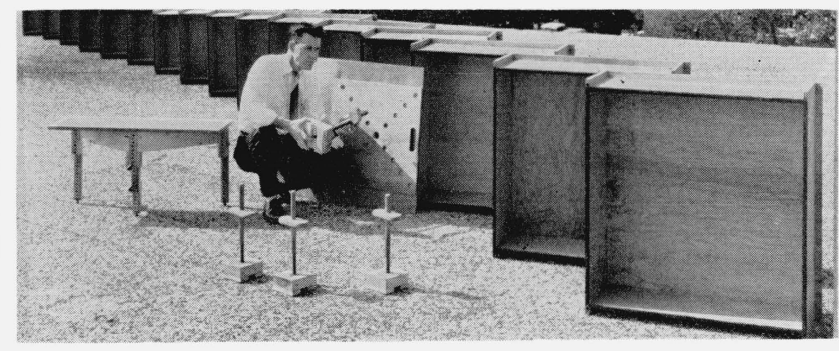

Figure 5.

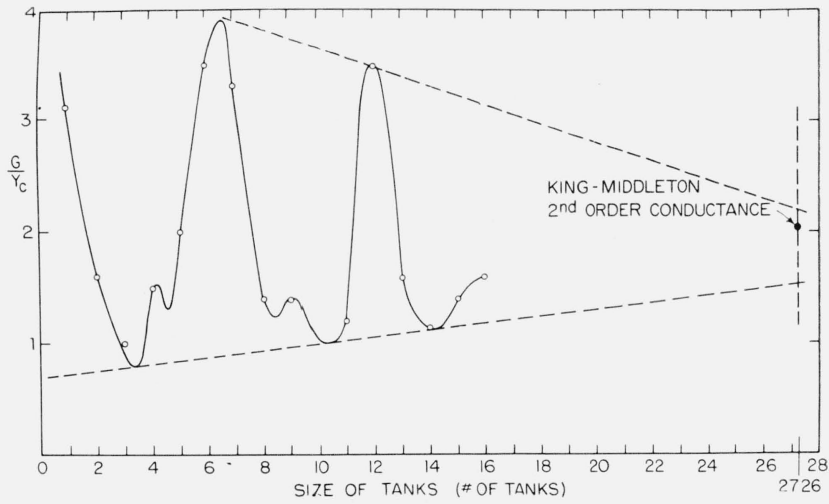

FIGURE 6a. Driving point conductance with various sizes of tanks).

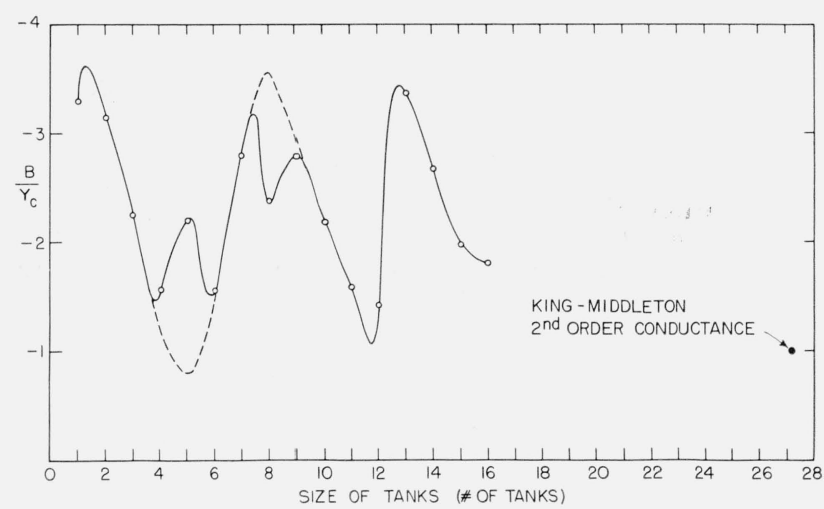

FIGURE 6b. Driving-point susceptance with various sizes of tanks.

The dimension $L$ of the smaller tanks was fixed at $L=1.0 \lambda_{w}$ and their width $D$ varied from $0.953 \lambda_{w}$ to $3.10 \lambda_{w}$ at intervals of $0.133 \lambda_{w}$.

The measured normalized driving-point admittance of an antenna with $\beta h=1.78$ and $\Omega=2 \mathrm{ln}$ $\frac{2 h}{a}=6.3$ and $\frac{\sigma}{\omega \epsilon_{r} \epsilon_{0}}=0.0354$ is shown in figures $6 \mathrm{a}$ and $6 \mathrm{~b}$ as a function of the tank number. In figure $6 \mathrm{a}$ a point of convergence of the oscillation in the normalized conductance is determined very roughly and rather arbitrarily by extending the envelopes of the extrema. The main tank corresponds to number 27.3 and the indicated variation in conductance is of the order of magnitude of \pm 10 
percent at this point. A comparable variation in the susceptance shown in figure $6 \mathrm{~b}$ should be obtained, but due to a resonance phenomenon discussed later, even a rough estimate of the percentage variation from the 16 measured points is meaningless.

\section{Significance of the Distance Between the Image Plane and the Back Wall}

In order to study the effect of the reflection from the back wall on the driving-point admittance, the solution at the back of the tank was replaced by two blocks of polyfoam. The distance $L$ was then varied from $L=1.017$ to $L=2.296$ by cutting slices from the polyfoam at intervals of $0.133 \lambda_{w}$. The measured driving-point admittances of antennas with $\beta h=1.78$ and $\beta h=3.57, \Omega=6.3$ in a solution with $\epsilon_{r}=78$ and $\frac{\sigma}{\omega \epsilon_{\tau} \epsilon_{0}}=0.0354$ are shown in figures $7 \mathrm{a}$ and $7 \mathrm{~b}$. It is seen that as the dimension $L$ is increased the admittance curves spiral inward on the Smith chart. As the length of the antenna is increased beyond $\beta h=\pi$, the electric field at small angles from the axis and, hence, in the direction of the back wall becomes relatively greater. It follows that the driving-point admittance for an antenna

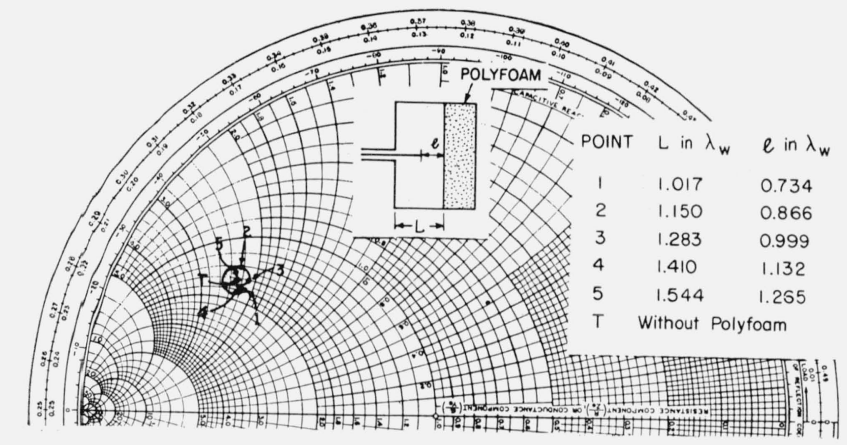

FIGURE 17a. Effect of back wall of the tank on the driving-point admittance of antenna with $\beta \mathrm{h}=1.78$ with different sizes of polyfoam wall.

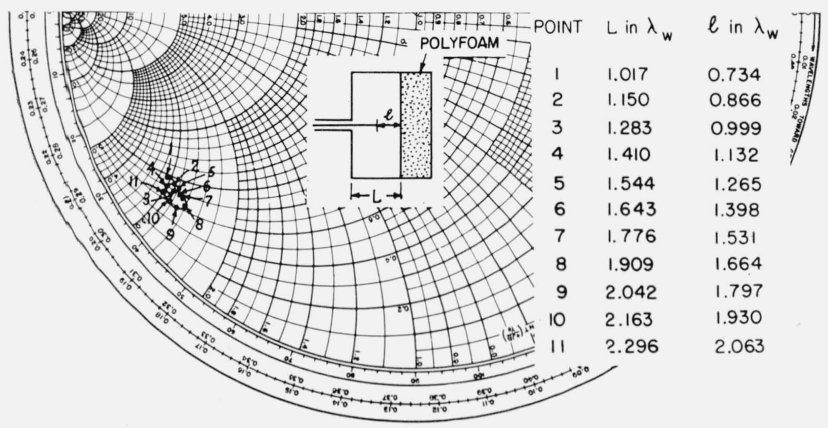

FIGURE 7b. Effect of back wall of the tank on the drivingpoint admittance of antenna with $\beta \mathrm{h}=3.57$ with different sizes of polyfoam wall. with $\beta h=1.78$ converges more rapidly as the back wall recedes than for an antenna with $\beta h=3.57$. The variation in the admittance of the antenna with $\beta h=1.78$ is less than 5 percent even at point number five $\left(L=1.54 \lambda_{w}\right)$. The variation in the admittance of the antenna with $\beta h=3.57$, however, does not decrease so rapidly and even at point number eleven $\left(L=2.30 \lambda_{w}\right)$ the variation is still approximately 4 percent to 8 percent. These results suggest that a limit for the length of an antenna in the measurement of its driving-point admittance in the main tank $\left(L=2.62 \lambda_{w}\right)$ is about $\beta h=3.57$, or possibly somewhat longer if considerable error can be tolerated.

\section{Installation of Antenna Off Center}

It can be anticipated that the effect of the reflection from the edges is in general reduced, if the antenna is installed off center. When the antenna is located at the center of a square ground plane the reflected waves that reach the antenna from the four side walls are equal so that the effect of one wall is multiplied by four. On the other hand, if the antenna is located off center the four reflected waves that reach the antenna are not equal, and they interfere at least partially.

An experiment to test this prediction was made with smaller tanks placed off center in the measurement of the driving-point admittance of the antenna. The center of the smaller tank was shifted horizontally from its original center by the distance $S$ at intervals of $0.133 \lambda_{w}$. It was not shifted in the vertical direction.

The admittance of one antenna was measured successively with tanks numbered 1,2 , and 5 . The results are shown in figures $8 b, 8 \mathrm{c}$, and $8 \mathrm{~d}$, respectively. The solution in the tank was the same as for the measurements discussed in the preceding section. The full line shows how the admittance moves on the Smith chart as the distance of the shift is increased. The broken line is a copy [Iizuka and King, 1962a] of the driving-point admittance with different sizes of smaller tanks shown in figure 8a. The admittance moves in the direction of the arrows as the size of the tanks is increased. It is interesting to note that the admittance in the full line always moves almost in the same direction as that of the arrow in the dotted line for the first few shifts in the position of the tank. In other words, the admittance always approaches the values measured with larger tanks provided the shift $S$ is less than a quarter wavelength. Thus, the shifting of the antenna from the center of the tank is effectively equivalent to enlarging the dimensions of the tank, and so demonstrates the advantage of installing the antenna off center in the main tank.

\section{Resonance Phenomenon of the Tank}

Among the measured driving-point admittances obtained with the series of tanks of increasing size, the points with the tanks numbered five and eight in figure 8a do not fall on the spiral. The measure- 


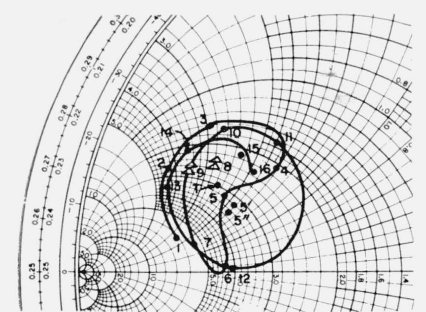

(a)

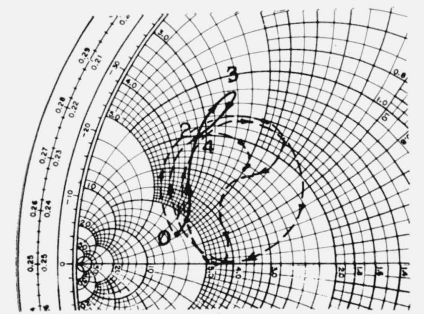

(b)

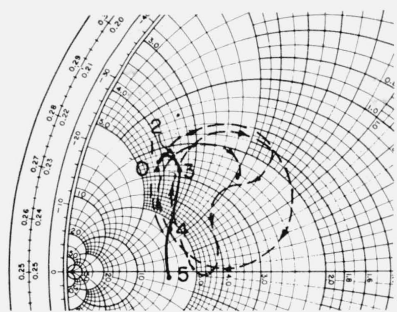

(c)

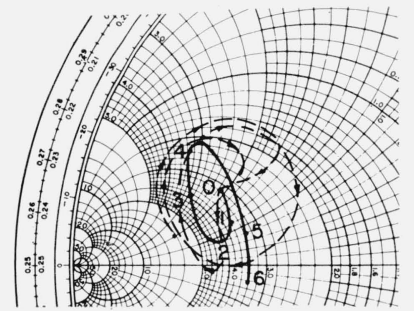

(d)

\begin{tabular}{|l|l|c|c|c|c|c|c|}
\hline Point & 0 & 1 & 2 & 3 & 4 & 5 & 6 \\
\hline$S$ in $\lambda w$ & 0 & 0.133 & 0.266 & 0.399 & 0.532 & 0.665 & 0.798 \\
\hline
\end{tabular}

Figure 8. Driving-point admittance with tanks of different sizes in $a$, with tank No. 1 installed off center in $b$, with tank No. 2 installed off center in $c$, and with tank No. 5 installed off center in d.

ments for these two points were repeated three times, but all measured values were in good agreement. In order to explain these deviations, the experimental results must be re-examined.

The curves of the conductance and susceptance in figures $6 \mathrm{a}$ and $6 \mathrm{~b}$ show not only a good periodic dependence on the size of the tank, but also certain peculiarities in the shapes and the relative positions of these two curves. To be more specific, the locations of the steepest slopes of the susceptance curve always coincide with the locations of the extrema of the conductance curve.

It is also noticed that there are two oscillations in the susceptance curve at the points five and eight and that both are located at the extrema of the curve, symmetric with respect to the locations of the steepest slope between points six and seven. Moreover, the driving-point admittances for these points are rather close together on the Smith chart. Furthermore, it has been verified that the distribution of the amplitude and phase of the current along a dipole antenna immersed in the tanks of these particular two sizes behave just as if the wavelength had become longer than in the tanks of all the other sizes [Iizuka and King, 1962a]. It appears possible to interpret these phenomena as the result of a condition of resonance with significant standing waves generated in the tank. In general, the wavelength at resonance is longer than that in free space, and the values of the driving-point admittance of the consecutive resonances are close together. The measured results correspond to this picture.

Another way of checking this interpretation is to observe the change in the field intensity inside the tank with respect to a change in the driving fre-

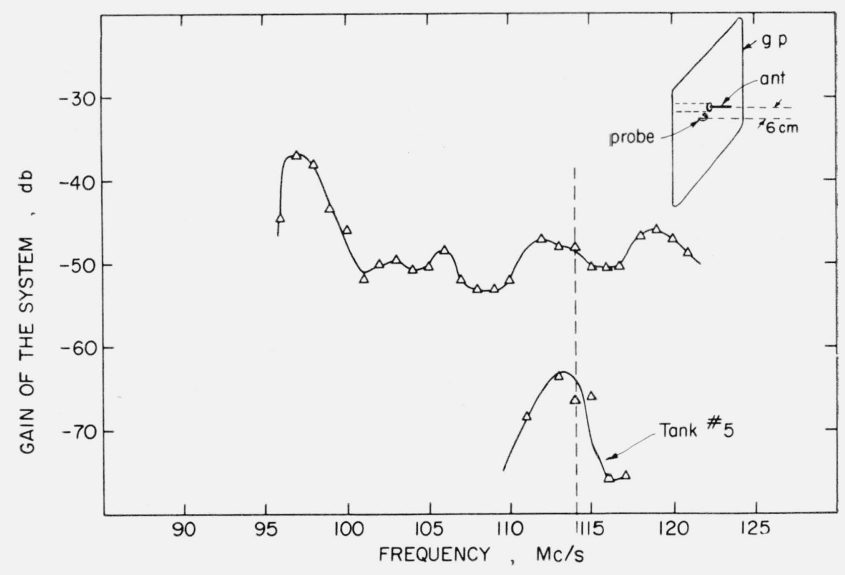

Figure 9. Frequency characteristics of the system.

quency. For this purpose an additional shielded probe was installed on the image plane $0.2 \lambda_{w}$ away from the antenna inside tank number 5 . The measured result is the lower curve in figure 9 . It is seen that as the frequency was varied from the original frequency of $114 \mathrm{Mc} / \mathrm{s}$ to either lower or higher values, the output from the probe decreased just as if the cavity had been detuned. The same measurement was made with the main tank to see whether it was off resonance. The upper curve in figure 9 is the result of this measurement; it shows that the main tank is not resonant at $114 \mathrm{Mc} / \mathrm{s}$.

Additional evidence can be found to confirm the condition of resonance of tank number 5 from a careful comparison of the curves in figures $8 b, 8 c$, and $8 \mathrm{~d}$ of the driving-point admittance as a function of the lateral shift in the position of the smaller tanks. Tanks numbered 1,2, and 5 were shifted in steps of $0.133 \lambda_{w}$. The difference between the admittance without shift and that with the first shift $(S=$ $0.133 \lambda_{w}$ ) may be compared in these three curves. It is seen that the curve for tank number 5 is the largest even though this was the largest of the three tanks ( $D$ for tanks 1,2 , and 5 are $0.95 \lambda_{w}, 1.09 \lambda_{w}$, and $1.49 \lambda_{w}$ respectively) and the fraction $S / D$ was the smallest. Evidently tank number 5 is most sensitive to a perturbation of the system, since it is resonant.

The studies in this section all indicate that a condition of resonance may be produced in a tank filled with a solution of relatively low conductivity. In designing the main tank it is necessary to verify that it is off resonance by checking with one or more of the above-mentioned methods. Note that a condition of resonance cannot be determined in a simple manner familiar from the theory of resonators with metal walls.

\section{Temperature Dependence of the Electrical Properties of the Solution}

The measurements of the driving-point impedance of the same length of antenna $(\beta h=1.78)$ were repeated three times at the water temperature $19.2{ }^{\circ} \mathrm{C}$ in the midsummer, $17.2^{\circ} \mathrm{C}$ in the fall, and 
$16.8{ }^{\circ} \mathrm{C}$ in the beginning of winter. All three measured values were practically the same.

A change of $4{ }^{\circ} \mathrm{C}$ in the temperature of the water brings about a change of 1.5 in the dielectric constant and a change of 1.6 and $10^{-3}$ in the value of

$\frac{\sigma}{\omega \epsilon_{\tau} \epsilon_{0}}$ at $18{ }^{\circ} \mathrm{C}$ and $300 \mathrm{Mc} / \mathrm{s} \quad\left(\epsilon_{r}=79.5\right.$ and $\frac{\sigma}{\omega \epsilon_{r} \epsilon_{0}}=$ 1.88 and $10^{-3}$ at $18{ }^{\circ} \mathrm{C}$ ) [von Hippel, 1958]. This fact may be used to reach the following conclusion: Since the driving-point admittance of an antenna is insensitive to the changes

$$
\begin{gathered}
\frac{\Delta \epsilon}{\epsilon}=\frac{1.5}{79.5}=0.019 \\
\frac{\Delta\left(\frac{\sigma}{\omega \epsilon_{r} \epsilon_{0}}\right)}{\frac{\sigma}{\omega \epsilon_{r} \epsilon_{0}}}=\frac{1.6 \times 10^{-3}}{18.8 \times 10^{-3}}=0.085
\end{gathered}
$$

an error of 2 percent in the measurement of $\epsilon_{r}$ and of 8.5 percent in the measurement of $\frac{\sigma}{\omega \epsilon_{r} \epsilon_{0}}$ is tolerable insofar as the measurement of the driving-point admittance in the solution with $\frac{\sigma}{\omega \epsilon_{r} \epsilon_{0}}=0.036, \epsilon_{r}=78$, is concerned.

\section{Conclusion}

It has been shown by a systematic series of measurements how the finite size of a dissipative medium may significantly affect antenna measurements. Of particular interest is the possible occurrence of resonances which are evidently possible even in very large tanks if the conductivity of the medium is not too high.

\section{References}

Iizuka, K. (1963a), An experimental study of the insulated dipole antenna immersed in a conducting medium, to appear in IRE Trans. Ant. Prop. AP-11.

Iizuka, K. (1963b), An experimental investigation on the behavior of the dipole antenna near the interface between a conducting medium and free space, Cruft Lab. Scientific Report No. 4, Harvard University, Cambridge, Mass.

Iizuka, K., and R. W. P. King (1961a), Apparatus for the study of the properties of antenna in a conducting medium, Cruft Lab. Scientific Report No. 1, Harvard University, Cambridge, Mass.

Iizuka, K., and R. W. P. King (1961b), An experimental study of the properties of antennas in a conducting medium, Cruft Lab. Scientific Report No. 2, Harvard University, Cambridge, Mass.

Iizuka, K., and R. W. P. King (1962a), The dipole antenna in a homogeneous conducting medium, IRE Trans. Ant. Prop. AP-10, 384-392.

Iizuka, K., and R. W. P. King (1962b), An experimental study of the half-wave dipole antenna immersed in a stratified conducting medium, IRE Trans. Ant. Prop. AP-10, 393-399.

Iizuka, K., and R. W. P. King (1962c), The dipole antenna as a probe for determining the electric properties of a stratified medium, IRE Trans. Ant. Prop. AP-10, 783-784.

King, R.W.P., and K. Iizuka (1963), The complete electromagnetic field of half-wave dipole in a dissipative medium, to appear in IRE Trans. Ant. Prop. AP-11.

King, R. W. P. (1961), Dipoles in dissipative media, Proc. Symp. Math., 199-241, Research Center, University of Wisconsin Press, Madison, Wis.

Ramo, S., and J. R. Whinnery (1953), Fields and waves in modern radio, 290-292 (John Wiley \& Sons, Inc., New York, N.Y.; Chapman and Hall, Ltd., London, England).

von Hippel, A. R. (1958), Dielectric materials and applications, 2d ed., 294-370 (Technology Press of M.I.T., Cambridge, Mass, and John Wiley \& Sons, Inc., New York, N.Y.).

(Paper 67D4-275) 